\title{
Canadian Jewish Heritage Material Repositories / Liste de référence de tous les dépôts du patrimoine juif du Canada
}


JEWISH HISTORICAL SOCIETY OF BRITISH COLUMBIA, Vancouver 6184 Ash Street, Vancouver, BC, V5Z $3 G 9$

Archivist: Alysa Routtenberg

Tel: 604-638-7286 | Museum: 604-257-5199 | archives@jewishmuseum.ca | http://www.jewishmuseum.ca

JEWISH HISTORICAL SOCIETY OF SOUTHERN ALBERTA, Calgary

1607-90th Ave. S.W., Calgary, AB, T2V $4 V 7$

Archives Director: Agi Romer Segal

Tel: 403-444-3171 | jhssa@shaw.ca | http://jhssa.org/

JAHSENA (Jewish Archives and Historical Society of Edmonton and Northern Alberta), Edmonton 10220-156 St., Suite 200, Edmonton, AB, T5P 2R1

Archivist: Colleen Paull

Tel: 780-489-2809 | jahsena@shaw.ca| http://www.jahsena.ca/jahsena_001.htm

JEWISH HERITAGE CENTRE OF WESTERN CANADA, Winnipeg

C116 - 123 Doncaster Street, Winnipeg, MB, R3N 2B2

Archivist: Andrew Morrison

Tel: 204-477-7460 ext. 7461 | jhc@jhcwc.org | http://www.jhcwc.mb.ca/

ONTARIO JEWISH ARCHIVES, Toronto

4600 Bathurst Street, Toronto, ON, M2R 3V2

Archives Director: Dara Solomon

Tel: 416-635-2883 ext. 187 | oja1 @ujafed.org | www.ontariojewisharchives.org/

BETH TZEDEC REUBEN AND HELENE DENNIS MUSEUM, Toronto

1700 Bathurst Street, Toronto, ON, M5P 3K3

Curator: Dorion Liebgott

Tel: 416-781-3511 | museum@beth-tzedec.org | http://www.beth-tzedec.org/museum/

OTTAWA JEWISH ARCHIVES, Ottawa

21 Nadolny Sachs Private, Ottawa, ON, K2A 1R9

Archivist: Saara Mortensen

Tel: 613-798-4696 ext. 260 | archives@jewishottawa.com | http://www.jewishottawa.org

LIBRARY AND ARCHIVES CANADA / BIBLIOTĖQUE ET ARCHIVES CANADA, Ottawa

395 Wellington Street, Ottawa, ON, K1A ON4

Archives search: http://www.collectionscanada.gc.ca/lac-bac/search/arch

Reference questions by phone: 1-866-578-7777 (toll-free in Canada and US)

Reference questions by email: https://www.bac-lac.gc.ca/eng/assistance-request-form

ALEX DWORKIN CANADIAN JEWISH ARCHIVES /

ARCHIVES JUIVES CANADIENNES ALEX DWORKIN, Montreal

1590 Avenue Docteur Penfield, Montreal, QC, H3G 1C5

Archives Director: Janice Rosen

Tel: 514-931-7531 ext. 2 | archives@cjarchives.ca | http://www.cjarchives.ca

JEWISH PUBLIC LIBRARY ARCHIVES / ARCHIVES DE LA BIBLIOTĖQUE PUBLIQUE JUIVE, Montreal

1 carré Cummings Square, 5151 Côte Ste-Catherine Rd., Montreal, QC, H3W 1M6

Archivist: Shannon Hodge | Archivist Emeritus: Eiran Harris

Tel: 514-345-2627, extensions 3015, 3000 | archives@jplmontreal.org | www.jewishpubliclibrary.org

MONTREAL HOLOCAUST MUSEUM ARCHIVES / ARCHIVES DU MUSÉE DE L'HOLOCAUSTE DE

MONTRÉAL, Montreal

1 Carré Cummings Square, 5151, Côte Ste-Catherine Road, Montreal, QC, H3W 1M6

Tel: 514-345-2605 | info@museeholocauste.ca| http://www.museeholocauste.ca 
JEWISH GENERAL HOSPITAL ARCHIVES /

ARCHIVES DE L'HOPITAL GÉNÉRAL JUIF, Montreal

3755 Chemin de la Côte Ste-Catherine Rd., A-200, Montreal, Qc, H3T 1E2

Archivist: Linda Lei

Tel: 514-340-822, ext. 3277 | legacy@jgh.mcgill.ca | http://www.jgh.ca/en/archives

SAINT JOHN JEWISH HISTORICAL MUSEUM ARCHIVES, Saint John

91 Leinster Street, Saint John, NB, E2L 1J2

Curator/Archivist: Katherine Biggs-Craft

Tel: 506-633-1833| sjjhm@nbnet.nb.ca| http://jewishmuseumsj.com 